\title{
Optimal Tooth Reduction for Veneer Restorations: A Case Report
}

\author{
Carlos A Jurado
}

\begin{abstract}
Bonding to enamel has been shown to provide reliable results, and thus conservative tooth reduction is key to the success of the ceramic bonded veneers. The diagnostic wax is the first available to evaluate disparities between current and ideal tooth measurements. The mock-up provides the patient with a physical perception of the size and shape of the proposed veneers. The use of reduction guides assists the clinician in evaluating the specific amount of tooth structured to be removed during the preparation. This article demonstrates a conservative approach to tooth preparation combining different tooth reduction guides. Long-term success of the restoration requires following well-defined protocols for restorative material selection, conservative tooth preparation, and bonding ceramic protocols.

Keywords: Dental prostheses, Esthetic dentistry, fixed prosthodontics, Prosthodontic management.

International Journal of Prosthodontics and Restorative Dentistry (2019): 10.5005/jp-journals-10019-1244
\end{abstract}

\section{BACKGROUND}

Optimal tooth reduction is a fundamental requirement for function, esthetics, and longevity in restorative dentistry, especially for all ceramic restorations. ${ }^{1}$ Satisfactory tooth preparation can provide uniform reduction and acceptable clearance to allow the necessary thickness of the final restoration without disrupting the periodontal health and esthetics. It is highly suggested that a conservative approach be used on any occasion possible as an alternative treatment. The ability to retreat the restored tooth needs to be considered by the clinician when choosing a conservative or aggressive restorative treatment, especially for young adult patients. ${ }^{2}$ Veneers are the most conservative fixed restoration because they require to only remove $25-50 \%$ of the tooth structure in comparison to full coverage crowns. ${ }^{3}$ Furthermore, conservative tooth reduction are mandatory to create ideal adhesion, because excessive buccal reduction can lead to compromised bond strength due to penetration of the dentin. ${ }^{4}$ It has been demonstrated that conservative restorative techniques relies on bonding and adhesive luting, because intact enamel provides the most reliable substrate for etched ceramic laminate veneers. ${ }^{5}$

Currently, it is possible to fabricate ultrathin ceramic veneers with a thickness of $0.1-0.5 \mathrm{~mm}$, which need to be bonded with minimal or no tooth preparation in order to improve the tooth position, color, and shape. ${ }^{6}$ Several ceramic options are available for the clinician such as lithium disilicate, feldspathic porcelain, feldspathic reinforced with leucite, and lithium disilicate reinforced with zirconia. These types of ceramic materials have high proportion of glassy matrix that produces highly esthetic results and excellent adhesion with resin cement when treated with hydrofluoric acid followed by silane application. ${ }^{7}$ High survival rates with low failure numbers have been found for ceramic veneers bonded to enamel. ${ }^{8}$

Tooth reduction guides are recommended to make uniform space for the restoration in order to avoid undesirable situations. For fixed dental prostheses, ideal contours are established with the diagnostic wax-up, including any modification in vertical dimension and orientation in the plane of occlusion. Typical reduction guides are fabricated with polyvinyl siloxane (PVS) putty impression material or thermoplastic sheet.
Clinica Digital Dentistry, AT Still University, Arizona School of Dentistry and Oral Health, Mesa, Arizona, USA

Corresponding Author: Carlos A Jurado, Clinica Digital Dentistry, AT Still University, Arizona School of Dentistry and Oral Health, Mesa, Arizona, USA, Phone: +1 (480) 248 8152, e-mail: carlosjurado@atsu.edu How to cite this article: Jurado CA. Optimal Tooth Reduction for Veneer Restorations: A Case Report. Int J Prosthodont Restor Dent 2019;9(3):99-103.

Source of support: Nil

Conflict of interest: None

\section{Case Description}

A female patient aged 25 years presented to the dental clinic with the chief complaint "I do not like my smile because my teeth are small and I have spaces" (Figs 1 and 2). The patient stated that she just finished the orthodontic treatment and she was referred to close the spaces with restorations. After detailed evaluation,



Fig. 1: Preoperative photograph

(c) The Author(s). 2019 Open Access This article is distributed under the terms of the Creative Commons Attribution 4.0 International License (https://creativecommons. org/licenses/by-nc/4.0/), which permits unrestricted use, distribution, and non-commercial reproduction in any medium, provided you give appropriate credit to the original author(s) and the source, provide a link to the Creative Commons license, and indicate if changes were made. The Creative Commons Public Domain Dedication waiver (http://creativecommons.org/publicdomain/zero/1.0/) applies to the data made available in this article, unless otherwise stated. 


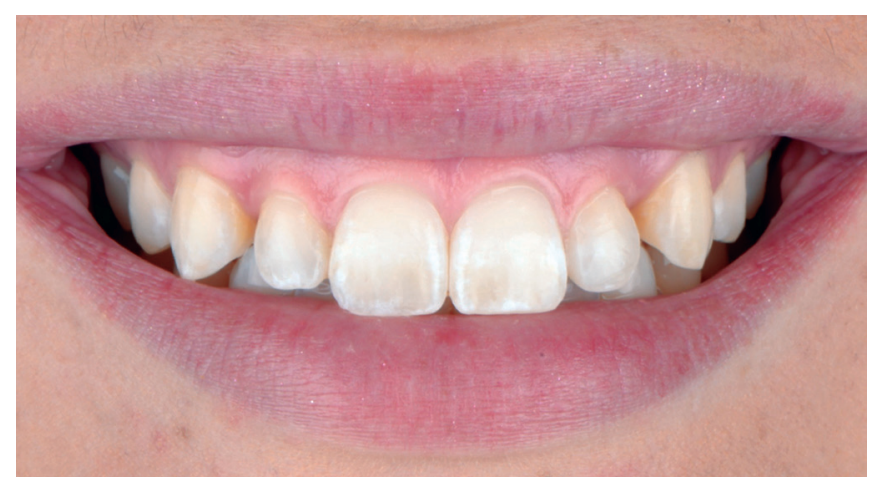

Fig. 2: Initial smile (upper dental)

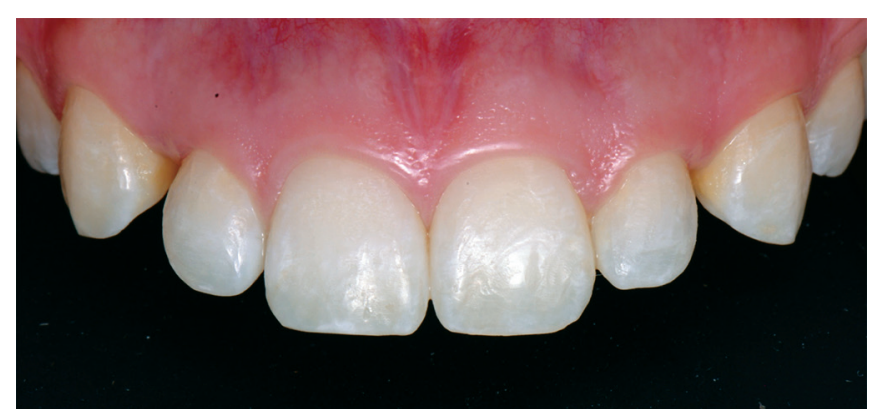

the diagnosis was excessive space between teeth \#6 to 7 and \#10 to 11 (Figs 3 to 5). Patient was informed of the need to perform a diagnostic wax-up (GEO Classic Renfert) followed by diagnostic mock-up with self-cured temporary composite material (Protemp Plus, 3M Espe) in order to evaluate the tentative future dimension of the restorations (Figs 6 and 7). The diagnostic mock-up was placed intraorally, and patient was pleased with the results and asked to move forward. The final treatment plan included lithium disilicate ceramic veneers on teeth \#6, 7, 10, and 11. Patient was explained that minimal tooth preparations will be performed in order to have

Fig. 3: Initial intraoral view

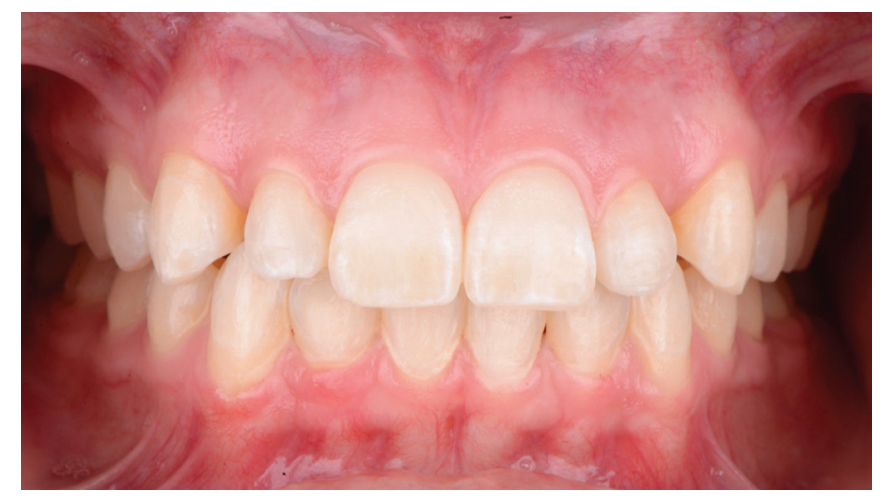

Fig. 4: Initial intraoral frontal view

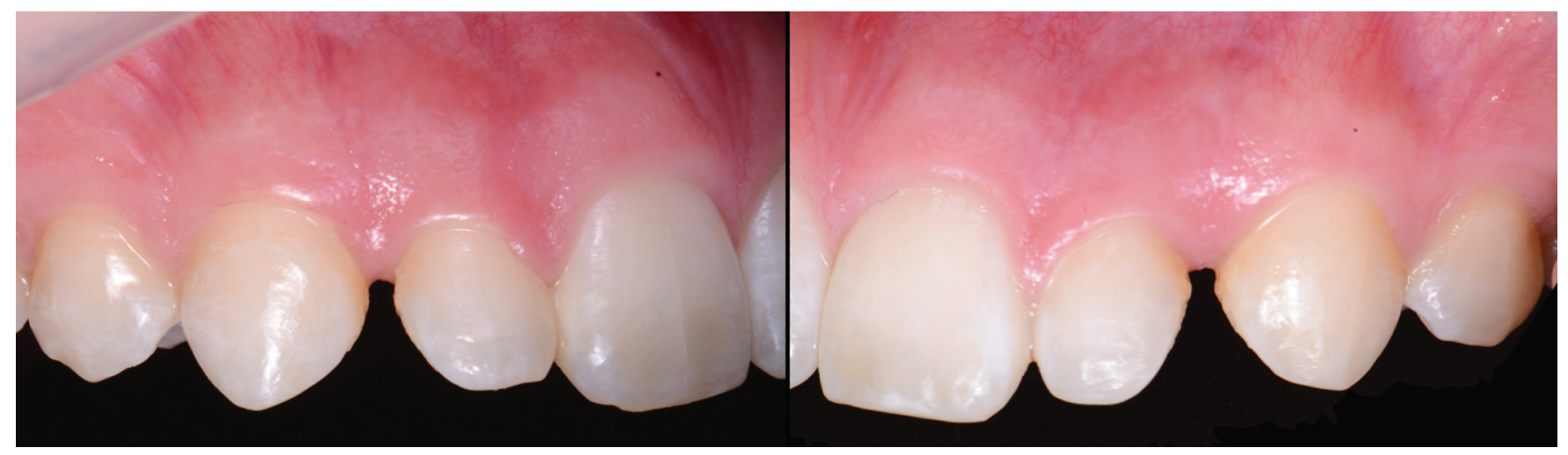

Fig. 5: Initial intraoral lateral views

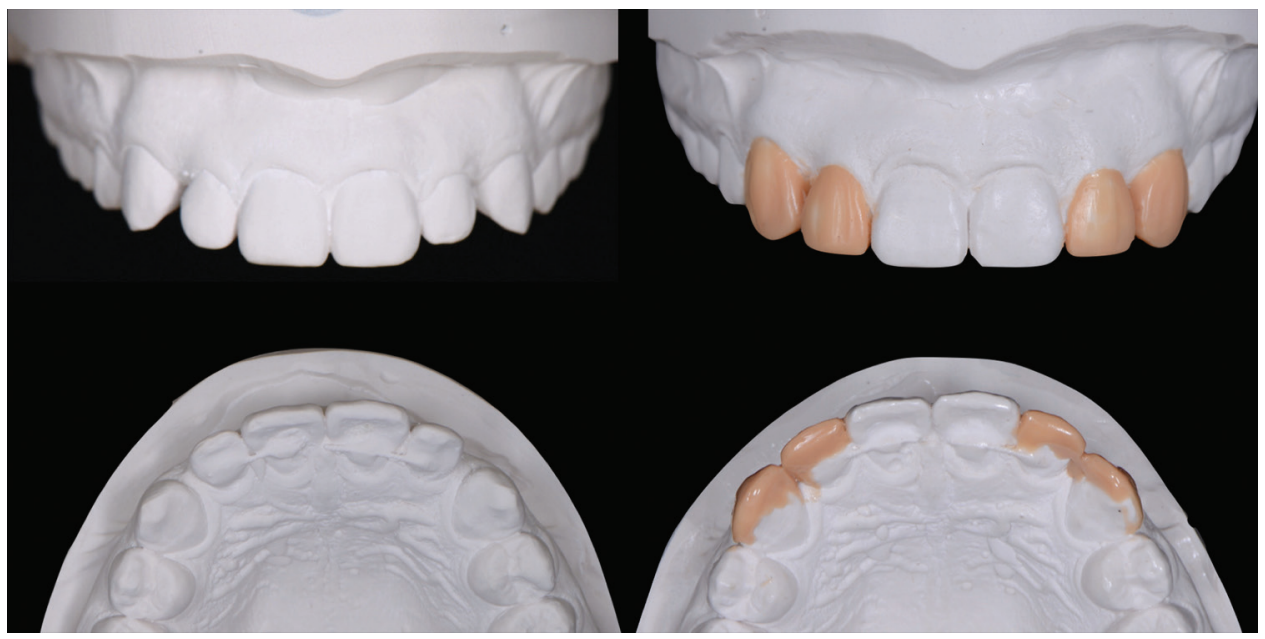

Fig. 6: Diagnostic wax-up 


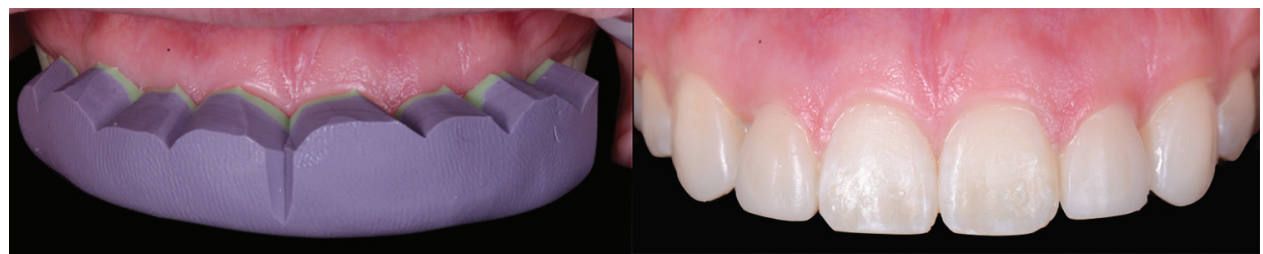

Fig. 7: Diagnostic mock-up
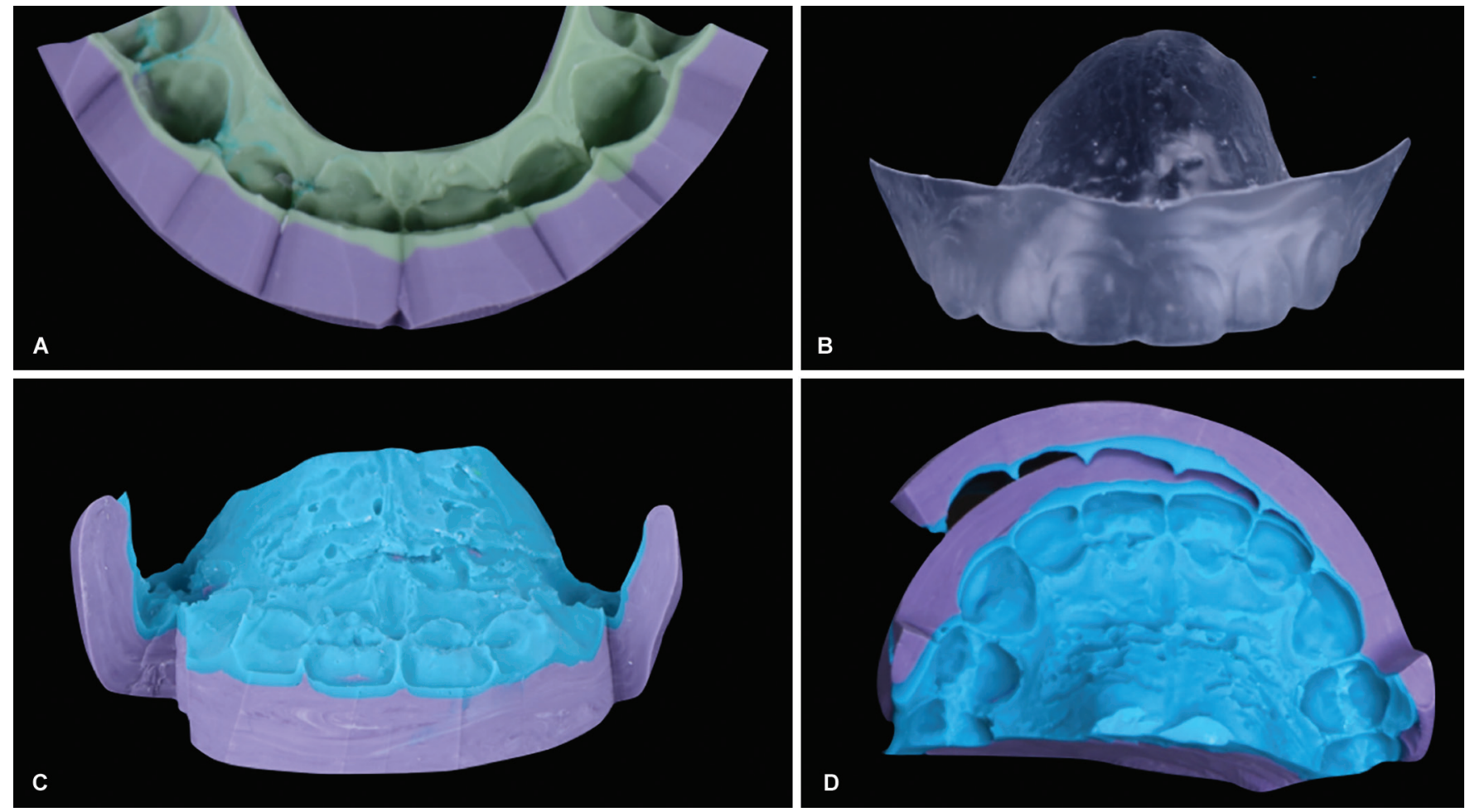

Figs 8A to D: Different types of guides used: (A) Putty guide for mock-up and provisional restorations; (B) Clear matrix for tooth preparation evaluation; (C) Incisal reduction putty guide; (D) Facial reduction putty guide

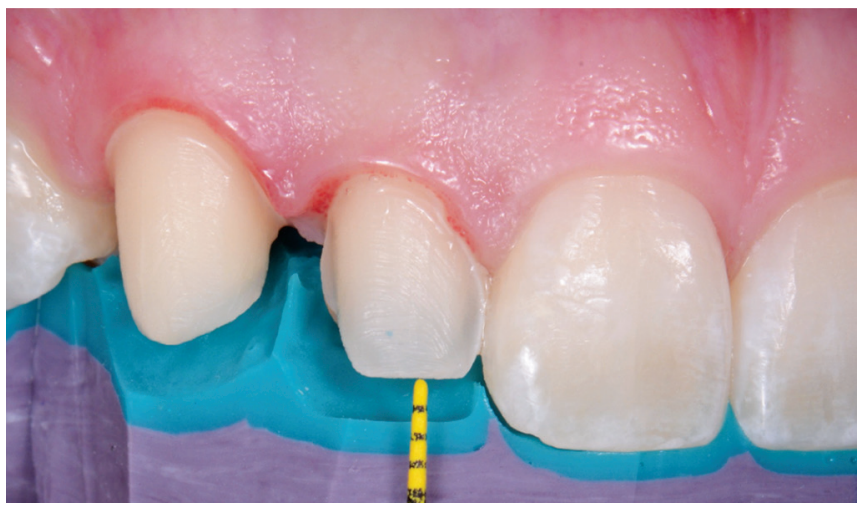

Fig. 9: Tooth reduction evaluation

the ideal space for the ceramic restorations. At the following clinical appointment, the same previously approved mock-up of self-cured material was created and placed in the patient's mouth and then horizontal and vertical depth grooves were cut on the teeth with a round diamond bur (801 Spherical, JOTA AG). Many reduction guides, based on the diagnostic wax-up, were fabricated; first the putty guide for provisional restorations (Fig. 8A), a putty guide to evaluate incisal reduction (Fig. 8B), a clear matrix guide to evaluate the overall preparations (Fig. $8 \mathrm{C}$ ), and finally a putty guide to evaluate the facial reduction (Fig. 8D) (Figs 8 and 9). The clear matrix also allowed an overall vision of the reduction, including areas not easily accessible, such as interproximal sites. Moreover, a putty reduction guide matrix (Hydrorise Putty, Zhermack SpA) was fabricated in order to evaluate incisal and facial reduction, and a periodontal probe was used to measure the reduction thickness. After minimal tooth reductions were completed, teeth were polished and smoothed, and corners were rounded using discs (Soft-Lex Discs, 3M ESPE), followed by sandblasting of the teeth with water and 20-micron aluminum oxide particles (AquaCare Aluminum Oxide Air Abrasion Powder, Velopex) (Fig. 10). A double-cord impression technique was used, first packing cord \#00 and then \#0 (Ultrapak, Ultradent Products Inc.), and the final impression was made using light body and heavy body consistency PVS (Virtual 380, Ivoclar Vivadent). Master cast and alveolar dies were fabricated with type IV stone (Fujirock, GC America Inc.). Ceramic veneers were fabricated out-of-lithium disilicate (IPS E.max Press $\mathrm{LS}_{2}$, Ivoclar Vivadent). The placement sequence of the ceramic restorations was first \#6 and 7, and then \#10 and 11 . The ceramic restorations received hydrofluoric acid surface treatment (IPS Ceramic Etching Gel, Ivoclar Vivadent) for 15 seconds, followed by rinsing and drying and then silane (Monobond-S, Ivoclar Vivadent) was applied for 60 seconds and then rinsed and dried. The tooth surface was first treated with $32 \%$ phosphoric acid gel (Uni-Etch w/BAC, Bisco Dental) for 20 seconds and then rinsed and gently dried. Then primer was applied (OptiBond FL, Kerr Dental), with the excess being removed by air, followed by light curing (VALO 

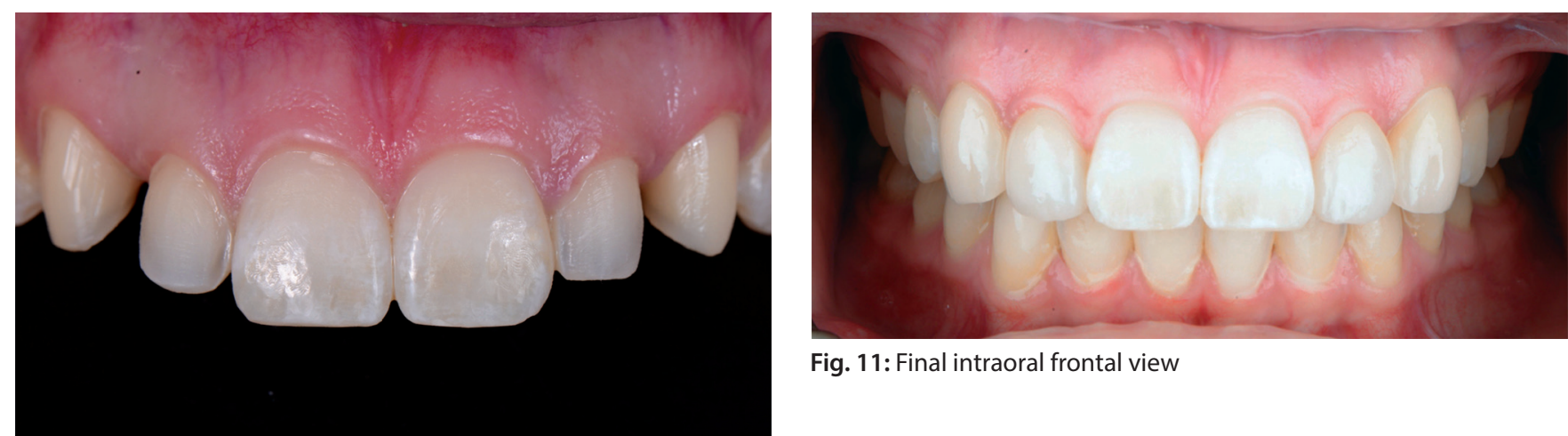

Fig. 11: Final intraoral frontal view

Fig. 10: Final tooth preparations

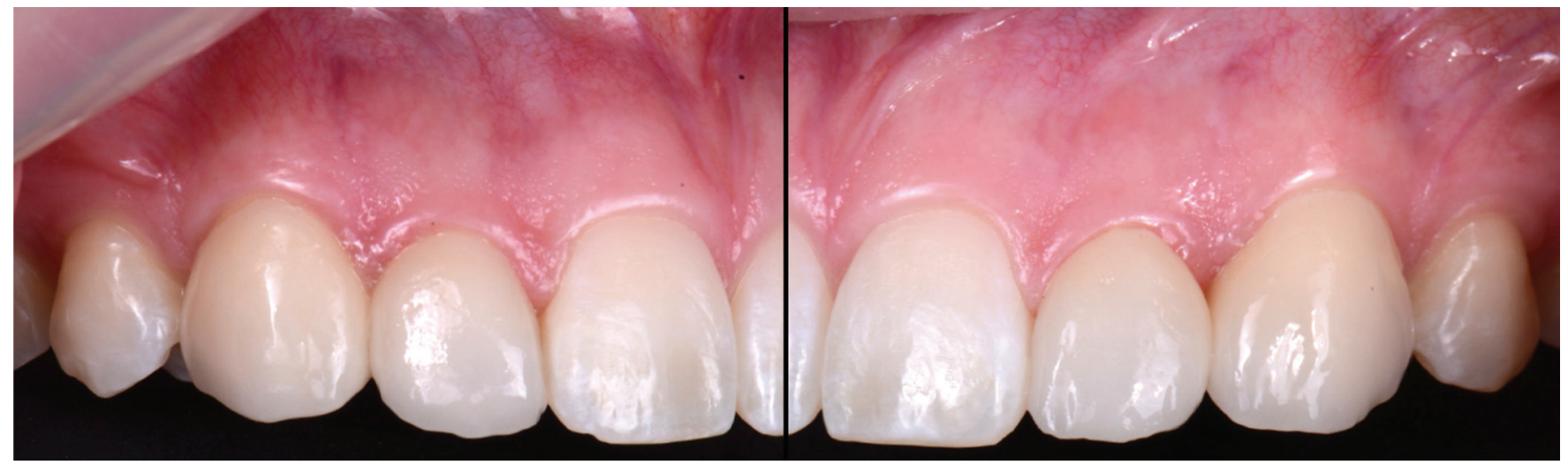

Fig. 12: Final lateral views

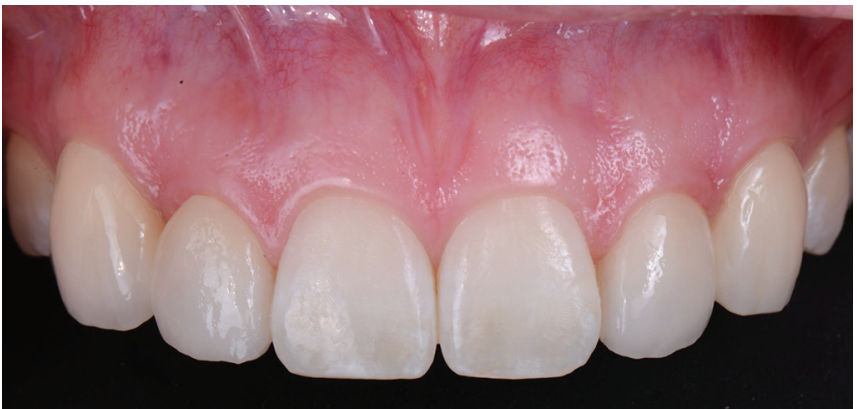

Fig. 13: Final intraoral

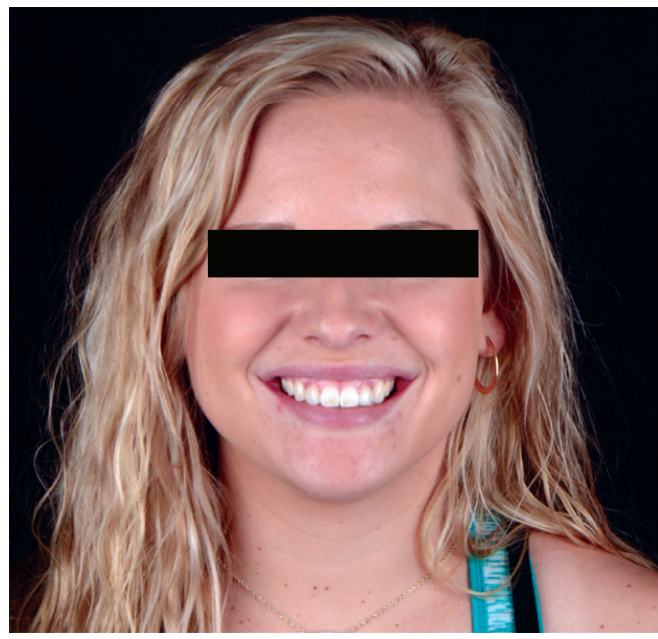

Fig. 15: Postoperative photograph

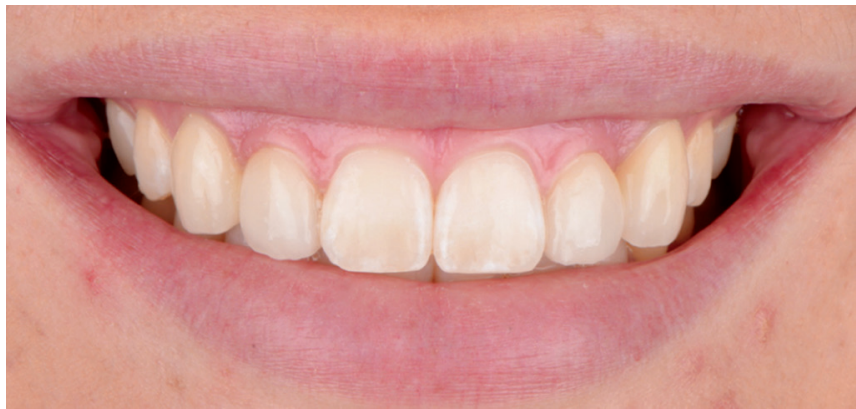

Fig. 14: Final smile (upper dental)

LED Curing Light, Ultradent Products Inc) for 20 seconds. The light color cement (Variolink Esthetic LC, Ivoclar Vivadent) was applied to the ceramic veneers, which were inserted onto the teeth, with the excess cement being removed with a micro brush and floss in the interproximal surfaces before light curing for 20 seconds on the facial, 20 seconds on mesial, 20 seconds on distal, and 20 seconds on the incisal surface. Glycerin gel was then applied to the ceramic surfaces in order to prevent an oxygen inhibition layer (Liquid Strip, Ivoclar Vivadent), and the surfaces were again light cured for 20 seconds each. Occlusion, excursive movements, and protrusion were checked. The patient was pleased with the final result (Figs 11 to 15). An occlusal device was provided to wear at night in order to prevent any damage to the restorations.

\section{Discussion}

This article describes how a properly planned diagnostic evaluation, thorough treatment planning, conservative tooth preparation, and 
ideal ceramic selection can fulfill a patient's high expectations. First, an additive diagnostic wax-up was made in order to perform the diagnostic mock-up. The wax-up information is transferred to the mouth and provides the patient an opportunity to experience a physical model of the proposed size, shade, and shade of the final veneers. ${ }^{9}$ Upon the patient's approval, several reduction guides can be fabricated based on the diagnostic wax-up. Some reduction guides are made with clear matrix that can provide the clinician with general evaluation of all prepared teeth. Another type of guides are fabricated with vinyl polysiloxane material and they are can provide measurements of individual areas such as incisal and facial. These reduction guides provide the restorative dentist with an opportunity to have a controlled-tooth reduction, appropriate to the type of ceramic material selected. Experienced clinicians may not need to use any type of reduction guide in order to achieve the minimal tooth reduction needed, but the author highly recommends them as clinician gets experience with veneer restorations. Having controlled tooth reduction can provide the optimal space for the final restorations fabricated by dental technician, milling manufacture, or conventional handcrafted. ${ }^{10}$ Furthermore, minimal tooth reduction can provide enough tooth structure left to prepare a full coverage crown in the future in case of failure of the ceramic restoration. Since there is no current dental prosthesis that can last forever, it should be considered for both the clinician and the patient to have a minimal and controlled tooth preparation.

\section{Conclusion}

The use of preparation guides provides the optimal space for adequate contour and thickness of the final ceramic veneers. The use of different reduction guides for the same teeth preparation will tremendously help the clinician to avoid over-or underpreparation and the fabrication of successful veneers. Conservative tooth preparation and appropriate adhesive systems should improve the longevity of the restorations.

\section{References}

1. Rosentiel SF, Land MF, Fujimoto J. Contemporary fixed prosthodontics, 4th ed., St. Louis: Elsevier; 2006. pp. 209-257.

2. Holm C, Tidehag P, Tillberg A, et al. Longevity and quality of FDPs: a retrospective study of restorations, 30,20 and 10 years after insertion. Int J Prosthodont 2003;16(3):283-289.

3. Edelhoff $D$, Sorensen J. Tooth structure removal associated with various preparation designs for anterior teeth. J Prosthet Dent 2002;87(5):503-509. DOI: 10.1067/mpr.2002.124094.

4. Ge C, Green CC, Sederstrom DA, et al. Effect of tooth substrate and porcelain thickness on porcelain veneer failure loads in vitro. $J$ Prosthet Dent 2018;120(1):85-91. DOI: 10.1016/j.prosdent.2017.10.018.

5. Peumans M, De Munck J, Fieuws S, et al. A prospective ten-year clinical trial of porcelain veneers. J Adhes Dent 2004;6(1):65-76.

6. Grandon F, Marcus N, Muster M. Esthetic rehabilitation with ultrathin ceramic veneers and direct mock-up in the treatment of dental erosion - a case report. J Oral Res 2019;7(6):254-259.

7. Spear F, Holloway J. Which all-ceramic system is optimal for anterior esthetics? J Am Dent Assoc 2008;139(Suppl):19s-24s. DOI: 10.14219/ jada.archive.2008.0358.

8. Gresnigt MMM, Cune MS, Jansen K, et al. Randomized clinical trial on indirect resin composite and ceramic laminate veneers: Up to 10-years findings. J Dent 2019;86:102-109. DOI: 10.1016/ j.jdent.2019.06.001.

9. Alberton SB, Alberton V, de Carvalho RV. Providing a harmonious smile with laminate veneers for a patient with peg-shaped lateral incisors. J Conserv Dent 2017;20(3):210-213. DOI: 10.4103/09720707.218311.

10. Farias-Neto A, de Medeiros FCD, Vilanova L, et al. Tooth preparation for ceramic veneers: when less is more. Int J Esthet Dent 2019;14(2): 156-164. 